

\section{Facharzt Pädiatrie}

\section{Große Medizin für kleine Knirpse}

Der Pädiater zählt zu den beliebtesten Fachärzten unter Medizinstudenten. Was viele unterschätzen: Die Behandlung eines kranken Kindes geht oft mehr an die Nieren als vergleichbare Fälle aus der Erwachsenenheilkunde. Trotzdem trägt die Kinderheilkunde das Attribut „Traumfach“ zu Recht - sofern man den richtigen Draht zur Klientel findet ...

$\mathrm{D}^{\mathrm{s}}$ VolkerOcker ist zufrieden. Alle Patienten aufder Intensivstation des Stuttgarter Olgahospitals sind versorgt. Es ist eine ruhige Nacht für den diensthabenden Oberarzt - bis ein zehnjähriges Mädchen mit Schüttelfrost, Fieber und Erbrechen eingeliefert wird. Sie leidet an einer Meningokokkenmeningitis und droht zu sterben, obwohl sie bereits hochdosiert Penicillin bekommt. Als ihr kleines Herz stehen bleibt, belebt sie Dr. Ocker

\section{So schnell Kinder krank werden, so} schnell werden sie oft wieder gesund.

\section{Von Minimaschinen und Waisenkrankheiten}

Diese beiden Verläufe zeigen: Freud und Leid liegen bei Pädiatern dicht beieinander. Kinderärzte müssen damit leben, dass ihre Patienten manchmal auch sterben - und für alle Beteiligten ist so ein Tod oft schlimmer als der eines Erwachsenen. Wahr ist aber auch: So schnell Kinder manchmal erkranken, so schnell und komplett werden sie oft wieder - insgesamt fünfmal in dieser Nacht. Er schließt sie an die Hämodialyse und die Hämofiltration an, um ihr Leben zu retten. Mit Erfolg: Morgens um 5.00 Uhr ist ihr Zustand stabil. Sechs Jahre später ist aus dem Mädchen eine junge Frau geworden, die ihre mittlere Reife macht. Sie hat die Hirnhautentzündung gut überstanden. Nur noch ein paar Narben an den Beinen erinnern an die Schreckensnacht - eine Folge der durch die septische Gerinnungsstörung verursachten Hautdefekte.

Ein anderer Tag, derselbe Ort: Wieder wird eine kleine Meningitis-Patientin ins Olgahospital eingeliefert. Jetzt heißt der diensthabende Arzt Dr. Friedrich Reichert. Mit allen Mitteln der modernen Medizin behandelt er die Infektion - doch dieses Mal kommt alle Mühe zu spät. Die Kleine verstirbt einige Wochen später auf der Intensivstation an inneren Blutungen. Zuvor waren ihr im Rahmen eines Waterhouse-FriderichsenSyndroms nach und nach die Extremitäten abgestorben.

\section{VERLOSUNG}

\section{Fit for Kids!}

Für die Arbeit mit kleinen Patienten braucht man das richtige Händchen. Wir geben Ihnen die passenden Werkzeuge in dieselbigen und verlosen das Advance II Kinder Stethoskop und einen kindgerechten Giraffen-Reflexhammer der Firma DocCheck. Den theoretischen Unterbau

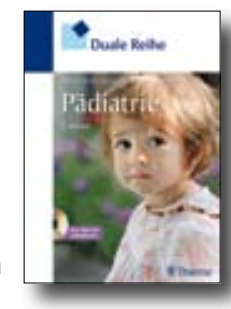

für Prüfung und Ernstfall können Sie sich mit einem von fünf Exemplaren der Dualen Reihe Pädiatrie anlesen. Teilnahme unter: www.thieme.de/viamedici/ zeitschrift/spezial, Stichworte „Untersuchung“ und „Pädiatrie“. Teilnahmeschluss: $\mathbf{6 . 8 . 2 0 1 2}$ auch wieder gesund - und belohnen einen damit für die $\mathrm{Ar}$ beit, die man investiert hat. Einige sind schon zwei Tage nach einer Herz-OP wieder fit und rennen über den Stationsflur.

Dass die freudigen Ereignisse bei der Therapie schwerkranker Kinder immer häufiger werden, ist ein Verdienst vieler neuer Therapieverfahren, die Pädiater in den letzten Jahren entwickelt haben. Ein Beispiel ist der künstliche Surfactant, mit dem extreme Frühchen behandelt werden können. Werden sie vor dem siebten Monat geboren, haben sie noch keinen Alveolen-Schutzfilm gebildet und erkranken häufig an einem infantilen respiratorischen Distress-Syndrom (IRDS). Der Surfactant wird über einen dünnen Katheter in die Luftröhre appliziert und danach der Patient per Maske beatmet. Oft kann so die belastende Beatmung mit Intubation vermieden werden. Ein weiterer Fortschritt ist die Miniaturisierung der Herz-Lungen-Maschinen: Diese in der Kinderherzchirurgie eingesetzten Geräte passen sich an die Blutvolumina von Neu- oder Frühgeborenen an. Zuvor mussten die Chirurgen auf Erwachsenengeräte zurückgreifen. Mit fatalen Folgen: Nachdem das kindliche Blut die große Maschine durchlaufen hatte, erkannte es der Körper oft nicht mehr als eigen an und reagierte mit einer unspezifischen Entzündung.

Das Stuttgarter Olgahospital hat als Kinderkrankenhaus der Maximalversorgung viele solche „Hightech-Features“ in petto - und Ärzte, die damit umgehen können. Daher kommen in die Klinik oft auch Kinder, die an sogenannten „Orphan Diseases“ leiden - seltene Krankheiten, deren Behandlung spezielle Fähigkeiten erfordert. Dazu zählen Erbkrankheiten wie Kinderrheuma oder das chronisch-infantile neuro-kutaneo-artikuläre Syndrom (CINCA), ein Gendefekt, der zu einer erhöhten Produktion von Interleukin-1 $\beta$ führt. Die Patienten leiden von Geburt an unter grippeähnlichen Symptomen und entwickeln oft Nierenschäden oder Hör- 
Husten oder leichtem Fieber in die Ambulanz. Trotzdem muss man den Sorgen der Eltern gerecht werden und ihnen ruhig erklären, dass dem Kind quasi nichts fehlt und es bald wieder gesund wird. Das verschiebt für Dr. Reichert die Prioritäten und hält ihn von der Niederlassung ab. Er schätzt, dass von zehn Kindern, die in den Notdienst kommen, nur eins oder zwei

\section{Der Leistungsdruck in den Schulen führt zu psychischen Problemen.}

gut lernen kann man im niedergelassenen Bereich, wie eine Kinderarztpraxis als primäre Anlaufstation funktioniert, wie man Patienten chronologisch versorgt und wie man entscheidet, ob man selbst behandeln kann - oder an einen Spezialisten überweisen muss. Deswegen setzt sich der Bundesverband der Kinder- und Jugendärzte e.V. (BVKJ) dafür ein, dass Teile wirklich krank sind und auf Station bleiben müssten. „Die oft unnötigen Arztbesuche haben viel mit der Verunsicherung durch die Gesellschaft und die Medien zu tun“, sagt er.

Diesen Eindruck bestätigt auch Dr. Gudrun Jacobi: „Der Instinkt zur Selbstheilung ist etwas verloren gegangen“, sagt die niedergelassene Kinderärztin. Sie sieht es deshalb als zentrale Aufgabe von Pädiatern an, das Bewusstsein der Eltern für einfache Methoden zu schärfen: Kalte Wickel, Ruhe und Zuwendung würden oft reichen, um einen fiebrigen grippalen Infekt auszukurieren. Auch in anderen Bereichen ihrer Tätigkeit schwört Dr. Jacobi auf eine Medizin, die die kleinen Patienten nicht isoliert, sondern eingebettet in ihren sozialen Kontext betrachtet. Das gilt z. B., wenn sie das Hör- und Sehvermögen oder die Sprachentwicklung von Kindern überprüft. Da sie im Bereich der Neuropädiatrie spezialisiert ist, hat die Ärztin eine eigene „Kopfschmerzsprechstunde“ - in der sie immer darauf achtet, ob eine neurologische Ursache vorliegt oder ob etwas anderes dahintersteckt: „Wird ein Elternteil arbeitslos, ist ein Familienmitglied psychisch oder körperlich krank oder gibt es Stress mit den Mitschülern, können dies Ursachen für Kopfschmerzen, Schulangst oder Schlafstörungen sein“, erklärt sie.

\section{Pädiatrische Baustellen}

Solche Hintergründe zu erfassen, kann man als angehender Kinderarzt besonders gut in einer Praxis erlernen, wo man die Patienten über Jahre begleitet und die Familien gut kennt. Auch

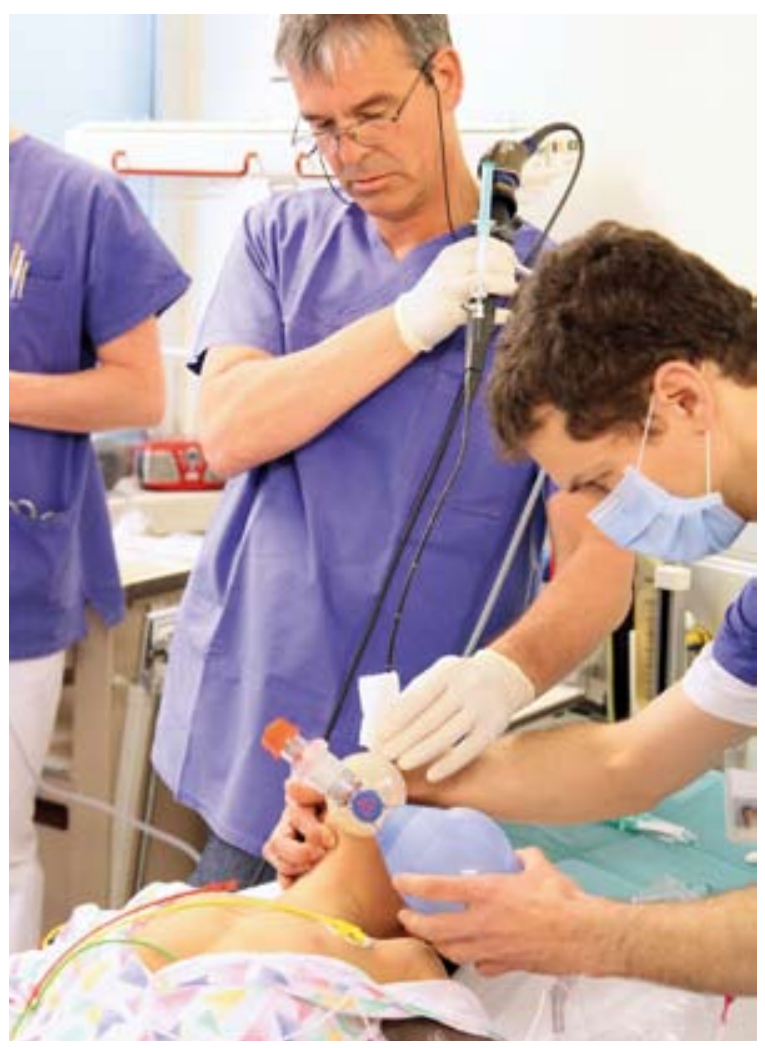

der Ausbildung in Zukunft auch in Kinderarztpraxen absolviert werden können. Das ist heute schon in Einzelfällen möglich, aber eher die Ausnahme, weil niedergelassene Pädiater die Weiterbildung selbst zahlen müssen. „Das ist ungerecht“, erläutert Dr. Klaus Rodens, Vorsitzender der BVKJ. „Schließlich werden allgemeinärztliche Weiterbildungen mit etwa 3.500 Euro pro Monat unterstützt.“

Das ist nicht die einzige Herausforderung, der sich die Organisatoren der pädiatrischen Weiterbildung in den nächsten Jahren stellen müssen: Heiß diskutiert wird z.B. auch die Frage, welche Konsequenzen sich aus der Subspezialisierung in der Pädiatrie ergeben. Pädiater bilden sich zunehmend zu Kinderkardiologen, -diabetologen oder -endokrinologen fort (Kasten). Das verbessert zwar die Versorgungsqualität - die Diagnose wird schneller gestellt und die Behandlung rascher eingeleitet. Aber nicht jede Ausbildungsstätte hat die Ausstattung, um die immer anspruchsvolleren Vorgaben an eine spezialisierte Weiterbildung zu erfüllen - was es für Assistenzärzte schwieriger macht, Teilkompetenzen zu erwerben.

Eine weitere Baustelle des BVKJ sind die U-Vorsorgeuntersuchungen. Sie dienen dazu, Fehlentwicklungen bei Kindern früh zu erkennen. Die bislang gängigen Untersuchungen U1 bis U9 decken alle Check-ups und Impfungen vom Baby bis zum Vorschulkind ab. Zudem gibt es die J1-Untersuchung für jüngere Teenager. Der Verband setzt sich dafür ein, dass nun auch die Vorsorgeuntersuchungen U10, U11 und J2 von allen

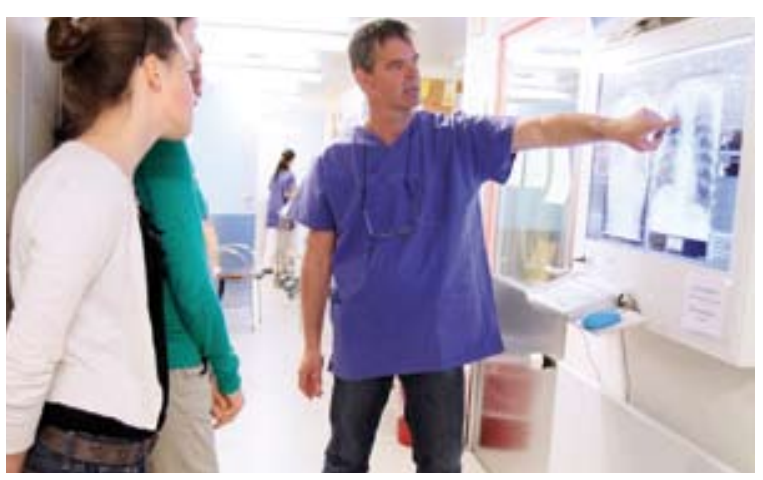

Pädiater mit Subspezialisierungen haben oft einen langen Arbeitstag: Doch Dr. Ocker lässt sich davon nicht in Hektik versetzen. Egal, ob er eine Bronchoskopie durchführt oder Studenten Röntgenbilder erklärt - er nimmt sich die Zeit, die er braucht.

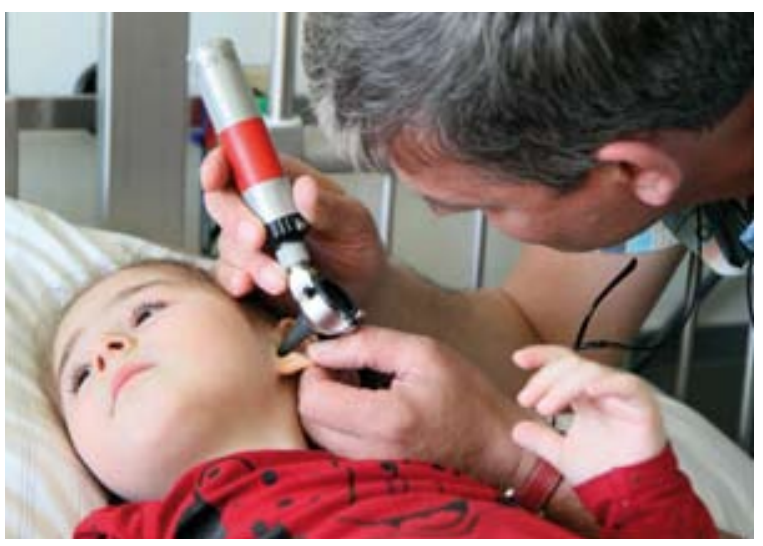

Ein kurzer Blick in den Gehörgang gibt Aufschluss, ob mit dem Trommelfell der Kleinen alles in Ordnung ist. 
